\title{
Two-stage revision surgery for periprosthetic joint infection following total hip arthroplasty
}

\author{
Timothy J. Fowler ${ }^{1}$, Adrian Sayers ${ }^{1}$, Michael R. Whitehouse ${ }^{1,2}$ \\ ${ }^{1}$ Musculoskeletal Research Unit, Translational Health Sciences, Bristol Medical School, Southmead Hospital, Bristol, UK; ${ }^{2}$ National Institute for \\ Health Research Bristol Biomedical Research Centre, University Hospitals Bristol NHS Foundation Trust, University of Bristol, Bristol, UK \\ Correspondence to: Mr. Timothy J. Fowler, MRCS. Musculoskeletal Research Unit, Bristol Medical School, Learning \& Research Building, Southmead \\ Hospital, Southmead Road, Bristol BS10 5NB, UK. Email: t.j.fowler@bristol.ac.uk. \\ Provenance: This is an invited article commissioned by the Academic Editor Dr. Ziming Chen (Centre for Orthopaedic Translational Research, \\ Medical School, University of Western Australia, Nedlands, Australia). \\ Comment on: Petis SM, Abdel MP, Perry KI, et al. Long-Term Results of a 2-Stage Exchange Protocol for Periprosthetic Joint Infection Following \\ Total Hip Arthroplasty in 164 Hips. J Bone Joint Surg Am 2019;101:74-84.
}

Submitted Dec 02, 2018. Accepted for publication Dec 20, 2019.

doi: 10.21037/atm.2019.12.126

View this article at: http://dx.doi.org/10.21037/atm.2019.12.126

\section{Introduction}

Total hip arthroplasty (THA) is a common, highly successful surgical intervention for the treatment of symptomatic degenerative conditions of the hip, such as osteoarthritis (1). Periprosthetic joint infection (PJI) is an uncommon, but potentially devastating complication of THA, with a reported incidence of between $0.6 \%$ and $2.2 \%$ (2-4). PJI often necessitates invasive treatment strategies, such as revision surgery, and has a significant negative impact on patients' quality of life $(5,6)$. With more than 1 million THAs performed worldwide each year, the health and economic burdens of this infective complication are substantial (1,3). Furthermore, two large national registry studies have demonstrated that the risk of revision due to hip PJI is rising $(7,8)$. In this context, an understanding of the most effective treatment options is of great importance.

The three principal treatment options for hip PJI include: surgical debridement with antibiotics and implant retention, with or without modular exchange (DAIR); one-stage revision; or two-stage revision. In contemporary healthcare settings, the additional options of excision, or amputation are considered a last resort. DAIR is commonly used in acute PJIs involving bacteria with known sensitivities, wellfixed prostheses and in patients unfit for major revision surgery. However, patients who undergo DAIR may require lifelong suppressive antibiotics and approximately half will subsequently require revision with implant exchange $(9,10)$.
Revision for hip PJI involves removal of the existing prosthesis, debridement of affected tissues, administration of antibiotics and reimplantation of a new prosthesis. 'Onestage' revision refers to the completion of this protocol in a single operation under one anaesthetic, whereas a 'two-stage' approach refers to the delayed reimplantation of a definitive THA, following completion of a course of sensitivityguided antimicrobial therapy. Two-stage protocols often utilize a temporary prosthesis, or antibiotic spacer prior to the definitive second stage.

\section{Summary of paper}

Petis et al. have recently published the results of a singlecentre series of 164 THAs in 162 patients, who underwent a two-stage surgical revision for PJI (11). They report the long-term re-infection and mechanical failure rates in a selective cohort, which has a mean follow-up of 12 years (range, 2 to 21 years). The paper offers a valuable contribution to the existing literature on this subject and we commend the authors for establishing such long-term outcomes, which are very scarce in this population.

The authors included adult patients who underwent both stages of revision at their institution (i.e., patients who underwent the first stage at another centre were excluded). All patients received an antibiotic spacer. The mean duration of antibiotics was 8 weeks, and the mean duration 
between cessation of antibiotics and the second stage was 7 weeks. Patients were excluded if they had received any prior treatment for infection of the joint in question. This study is novel in that, to the authors knowledge, no previous study reporting the results of a two-stage revision protocol for PJI has excluded patients on the basis of prior treatment for infection.

The principal finding of this study was an overall rate of recurrence of infection of $15 \%$ up to 15 years after THA. There were no additional episodes of reinfection between 10 and 15 years. The authors note that this 15 -year reinfection rate is similar to that observed in similar shorter-term reports of two-stage revision for PJI (12). The authors attribute this relatively high success rate to the "pure" cohort of patients who have had no prior treatment for infection. This is a reasonable suggestion, which could be quantified through direct comparison with a parallel "impure" cohort. However, it is important to highlight that both primary and revision THAs were included and grouped together in this cohort; given primary and revision THAs have different survival profiles in terms of revision for PJI (7), one might argue that this undermines the "purity" of the cohort.

Other key conclusions of this paper include: the utilization of chronic antibiotic suppression was predictive of re-infection; aseptic mechanical failure was lower than previously reported; clinical outcomes (measured by Harris hip score) improved significantly after revision and this improvement was sustained throughout follow-up; and hip instability was a common complication (12\%), which surgeons should be prepared to mitigate and manage operatively. With regards to chronic antibiotic suppression predicting the recurrence of infection, which is likely to be confounded by indication, the authors appropriately suggest that this observation may be due to suppression being used selectively in patients deemed to be at high risk of recurrence. They attempted to account for this in their modelling by the use of propensity score matching, but as the precise reason why patients were selected for chronic suppression was not available, or may be multifactorial, the matching on the basis of the factors described is not likely to fully account for the selection effect, and residual confounding may persist leading to the observed association.

The authors also use competing risk (CR) models to calculate the cumulative incidences of re-infection, aseptic and all-cause revisions. They say that Kaplan-Meier (KM) analysis, as used in previous studies on this subject, can 'overestimate treatment success when a death rate exists in a cohort'. They go on to say that the use of CR models provides ' $a$ more accurate estimation of the true success of twostage exchange'. This is a contentious topic, which is a source of ongoing debate in the arthroplasty literature (13), and it is important for the reader to understand in which context each method is applicable. Commonly used methods of survival analysis, such as the KM method and Cox regression models, estimate net failure and depend solely on the predefined event of interest (in this case re-infection, or mechanical failure). In contrast, CR models estimate crude failure and depend on both the profile of the event of interest and other outcomes that have been considered $a$ priori to be competing events (in this case, death). In order to justify the use of CR models, the research question needs to be clearly framed in terms of the desired outcome, i.e., crude failure. The KM method was specifically designed to account for incomplete observations that arise due to episodes of non-informative censoring, such as death (14). We have explored this notion in detail in a recent article by Sayers et al. (13), in which Sayers argues in favour of the use of KM models in the context of arthroplasty failure. He states that, 'as far as we currently know, the mortality process is independent of whether implants are revised or not, i.e. mortality satisfies the non-informative censoring assumption of death (in the KM method)'.

The scenario in this paper by Petis et al. is complicated, in that they are not only analyzing the mechanical failure of hips revised for infection, but they were interested in the recurrence of infection. The association between reinfection following revision for PJI and mortality has not been fully explored; however, it is not unreasonable to assume an underlying association between the two. With this in mind, their belief that the use of CR methods will mitigate any bias induced by the mortality profile in this cohort is incorrect. The cumulative incidence function is a "what you see, is what you get" estimator, whereas net failure estimated using KM, describes the failure profile if everyone was to live until the end of follow-up. Neither the CR method or KM method can be said to be superior, they just tell you something slightly different things and different analyses may be needed in the same paper depending on the outcome of interest.

Aside from these methodological issues, their study has many strengths. The mean follow-up exceeds that of any published observational study on this subject. Furthermore, the number of participants is high; as of 2015 only 2 of 60 observational studies on this subject featured $>150$ 
patients (15). The conclusions of this paper are sensible and offer valuable insight into the longer-term outcomes of a two-stage revision protocol.

\section{Future research}

Going forward, the direction of research in this field is focussed on the comparison of one- and two-stage revision protocols. While two-stage revision is considered by many to be the gold standard in the treatment of PJI of the hip (16), there is growing popularity for the one-stage approach and a growing body of evidence to support its use. Onestage revision is becoming increasingly popular because, compared to two-stage revision, it has the potential to reduce the overall surgical burden on patients, and to reduce healthcare costs (17). In terms of re-infection rates, a comprehensive meta-analysis on this subject, which included 38 one-stage studies and 60 two-stage studies, has demonstrated no difference in re-infection rates for the two approaches (15). A more recent meta-analysis of pooled individual participant data from 1,856 participants with a median follow-up of 3.7 years, has indicated a hazard ratio for re-infection following two-stage revision of 1.7 when compared with one-stage revision and that variables such as the presence of a sinus, may not preclude single stage revision as previously believed (18).

To date, the available evidence from observational studies and systematic reviews on this subject has been inconclusive. Equipoise thus exists regarding the optimum treatment for patients suffering from PJI in THA, and this justifies the conduct of a prospective randomized controlled trial (RCT) (19). For example, the 'Infection: Orthopaedic Management' (INFORM) trial, is a multi-centre RCT coordinated by our unit, which aims to answer this question (20). The first patient was enrolled in March 2015 and it is anticipated that the results of this trial will be available in 2020 .

\section{Conclusions}

Petis et al. offer a valuable contribution to the literature on this subject and their study provides insight into the longer-term outcomes and clinical effectiveness of a twostage revision protocol for hip PJI. However, given the subtle methodological differences between CR methods and $\mathrm{KM}$ analysis, readers should take care when comparing the results with existing literature, or when communicating the results presented here to patients when shared decision making around treatment choices occurs.

\section{Acknowledgments}

This study was supported by the NIHR Biomedical Research Centre at University Hospitals Bristol NHS Foundation Trust and the University of Bristol. The views expressed in this publication are those of the author(s) and not necessarily those of the NHS, the National Institute for Health Research or the Department of Health and Social Care.

\section{Footnote}

Conflicts of Interest: The authors have no conflicts of interest to declare.

Ethical Statement: The authors are accountable for all aspects of the work in ensuring that questions related to the accuracy or integrity of any part of the work are appropriately investigated and resolved.

\section{References}

1. Ferguson RJ, Palmer AJ, Taylor A, et al. Hip replacement. Lancet 2018;392:1662-71.

2. Blom AW, Taylor AH, Pattison G, et al. Infection after total hip arthroplasty. The Avon experience. J Bone Joint Surg Br 2003;85:956-9.

3. Kurtz SM, Lau E, Watson H, et al. Economic burden of periprosthetic joint infection in the United States. J Arthroplasty 2012;27:61-5.e1.

4. Ong KL, Kurtz SM, Lau E, et al. Prosthetic joint infection risk after total hip arthroplasty in the Medicare population. J Arthroplasty 2009;24:105-9.

5. Moore AJ, Blom AW, Whitehouse MR, et al. Deep prosthetic joint infection: a qualitative study of the impact on patients and their experiences of revision surgery. BMJ Open 2015;5:e009495.

6. Kunutsor SK, Beswick AD, Peters TJ, et al. Health Care Needs and Support for Patients Undergoing Treatment for Prosthetic Joint Infection following Hip or Knee Arthroplasty: A Systematic Review. PLoS One 2017;12:e169068.

7. Lenguerrand $\mathrm{E}$, Whitehouse $\mathrm{MR}$, Beswick $\mathrm{AD}$, et al. Revision for prosthetic joint infection following hip arthroplasty: Evidence from the National Joint Registry. Bone Joint Res 2017;6:391-8.

8. Dale H, Fenstad AM, Hallan G, et al. Increasing risk of 
prosthetic joint infection after total hip arthroplasty. Acta Orthop 2012;83:449-58.

9. Romanò CL, Manzi G, Logoluso N, et al. Value of debridement and irrigation for the treatment of periprosthetic infections. A systematic review. Hip Int 2012;22 Suppl 8:S19-24.

10. Byren I, Bejon P, Atkins BL, et al. One hundred and twelve infected arthroplasties treated with 'DAIR' (debridement, antibiotics and implant retention): antibiotic duration and outcome. J Antimicrob Chemother 2009;63:1264-71.

11. Petis SM, Abdel MP, Perry KI, et al. Long-Term Results of a 2-Stage Exchange Protocol for Periprosthetic Joint Infection Following Total Hip Arthroplasty in 164 Hips. J Bone Joint Surg Am 2019;101:74-84.

12. Garvin KL, Miller RE, Gilbert TM, et al. Late Reinfection May Recur More Than 5 Years After Reimplantation of THA and TKA: Analysis of Pathogen Factors. Clin Orthop Relat Res 2018;476:345-52.

13. Sayers A, Evans JT, Whitehouse MR, et al. Are competing risks models appropriate to describe implant failure? Acta Orthop 2018;89:256-8.

14. Kaplan EL, Meier P. Nonparametric Estimation from Incomplete Observations. Journal of the American Statistical Association 1958;53:457-81.

Cite this article as: Fowler TJ, Sayers A, Whitehouse MR. Two-stage revision surgery for periprosthetic joint infection following total hip arthroplasty. Ann Transl Med 2019;7(Suppl 8):S261. doi: 10.21037/atm.2019.12.126
15. Kunutsor SK, Whitehouse MR, Blom AW, et al. ReInfection Outcomes following One- and Two-Stage Surgical Revision of Infected Hip Prosthesis: A Systematic Review and Meta-Analysis. PLoS One 2015;10:e0139166.

16. Cooper HJ, Della Valle CJ. The two-stage standard in revision total hip replacement. Bone Joint J 2013;95B:84-7.

17. Klouche S, Sariali E, Mamoudy P. Total hip arthroplasty revision due to infection: a cost analysis approach. Orthop Traumatol Surg Res 2010;96:124-32.

18. Kunutsor SK, Whitehouse MR, Blom AW, et al. One- and two-stage surgical revision of peri-prosthetic joint infection of the hip: a pooled individual participant data analysis of 44 cohort studies. Eur J Epidemiol 2018;33:933-46.

19. Moore AJ, Blom AW, Whitehouse MR, et al. Managing uncertainty - a qualitative study of surgeons' decisionmaking for one-stage and two-stage revision surgery for prosthetic hip joint infection. BMC Musculoskelet Disord 2017;18:154.

20. Strange S, Whitehouse MR, Beswick AD, et al. Onestage or two-stage revision surgery for prosthetic hip joint infection--the INFORM trial: a study protocol for a randomised controlled trial. Trials 2016;17:90. 